\title{
Study on flow noise characteristics of Bionic cylinder based on acoustic analogy
}

\author{
Zixian Cui ${ }^{1, *}$, Hao Song ${ }^{1}, Q i \mathrm{Li}^{1}$, Buchao $\mathrm{An}^{1}$, and Lin $\mathrm{Su}^{1}$ \\ ${ }^{1}$ Systems Engineering Research Institute, Fengxian east road No.1, Haidian district, Beijing, China
}

\begin{abstract}
The drag and noise reduction of the flow around a cylinder is one of the important topics in hydrodynamics and acoustics. In this paper, three typical bionic cylinders are designed based on the serrated structure on the surface of shark skin. Using Large eddy turbulence model and Lighthill's acoustic analogy method, the flow noise characteristics of smooth cylinder and three kinds of bionic cylinders at different Reynolds numbers were compared, and the structure of cylinder surface was optimized. The results show that the main source of the flow noise around a cylinder is dipole noise, which is caused by the periodic fluctuating pressure on the cylinder surface.The bionic cylinder can reduce the amplitude of the fluctuating pressure, improve the wake flow field and reduce the wake vorticity, so as to reduce the noise. Among the three kinds of bionic cylinder, V-shaped bionic cylinder has the best noise reduction effect, and the critical value of $\mathrm{S} / \mathrm{H}$ of $\mathrm{V}$-shaped cylinder is about 2.5. When $\mathrm{s} / \mathrm{h}>2.5, \mathrm{~V}$-shaped bionic cylinder has no effect of noise reduction.
\end{abstract}

\section{Introduction}

In 1989, the U.S. Navy Center for ocean systems [1] studied the drag and noise characteristics of a body of revolution with a striped film on its surface. Park [2] studied the distribution of velocity and shear stress in the groove, and found that the noise on the groove surface can be reduced by about $1 \%$. At the same time, some scholars take the owl as the bionic research object, because most kinds of owls can fly silently. Graham [3] proposed in 1934 that the reason why owls can fly soundlessly is that the serrated structure on the edge of wings can change the flow state of fluid and control the shedding of fluid vortices, thus reducing noise. Inspired by the sawtooth structure of owl wing leading edge, soderman [4] of NASA Ames Research Center added serrated structure to the leading edge of two different sizes of rotor and conducted noise reduction experiments. It was found that the saw tooth structure has good noise reduction performance, with a maximum noise reduction of $8 \mathrm{~dB}$. Kun Chen[5] studied the sound suppression mechanism of the eagle owl (Bubo bubo) by Stereo Microscope (SM), Scanning Electron Microscopy (SEM) and Laser Scanning Confocal Microscope (LSCM). Fangfang Liu[6] presented the hydrodynamic model of an undulating fin and analyzed the effect of propagating wave motions on the forces acting on the fin surface.

\footnotetext{
*Corresponding author: cuizixian202011@163.com
} 
Most of the above researches are about aerodynamic noise, but the research on reducing the underwater noise of cylinder is relatively less. Inspired by the idea of bionics, this paper improves the surface of the cylindrical shell by bionics. The large eddy simulation (LES) combined with Lighthill acoustic analogy method is used to solve the flow noise of the bionic cylindrical shell. The noise reduction characteristics and mechanism of the bionic cylindrical shell underwater are discussed, which can provide some guidance for the reduction of the flow resistance and noise around the cylinder.

\section{Flow calculation model of bionic cylinder}

In this paper, a smooth cylinder model and three bionic cylinder models are designed. The geometric models are shown in Figure 1. The ratio of length to diameter of cylinders is 2.5:1.

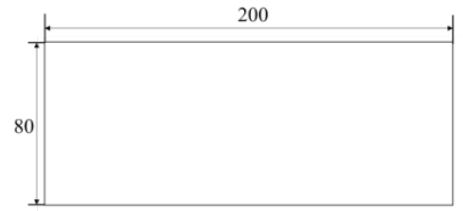

(a) Smooth cylinder

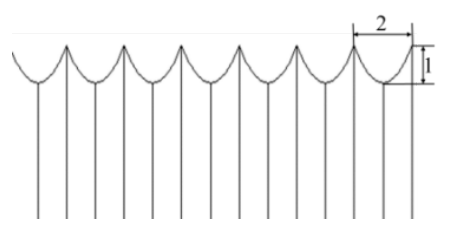

(c) U-shaped cylinder

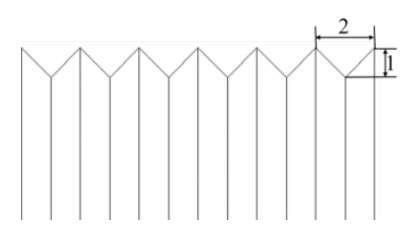

(b) V-shaped cylinder

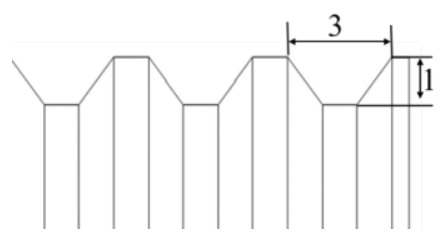

(d) Ladder shaped cylinder

Fig. 1. Geometric model of smooth cylinder and bionic cylinders.

\subsection{Flow field calculation}

\subsubsection{Flow field calculation model}

As shown in Figure 2, in order to ensure the calculation accuracy and save calculation resources, it is necessary to determine the appropriate calculation area. The calculation area is as follows, $\mathrm{D}$ is the diameter of the cylinder and $\mathrm{h}$ is the height of cylinder. The length of inflow section $\mathrm{L} 1=4 \mathrm{D}$, the length of outflow section $\mathrm{L} 2=15 \mathrm{~d}$, the width $\mathrm{Ld}=7 \mathrm{~d}$, and the height $\mathrm{H}=1.5 \mathrm{~h}$. A rectangular coordinate system is established. The center of the bottom surface of the cylinder is taken as the coordinate origin $\mathrm{O}$. The direction of the flow is $\mathrm{X}$-axis, The vertical flow direction is y-axis, and the development direction is z-axis.

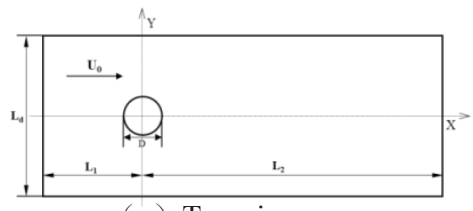

(a) Top view

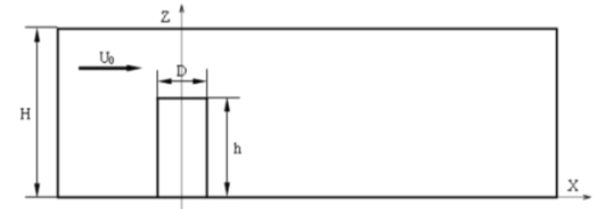

(b) Main view

Fig. 2. Geometric model of computational fluid domain. 
The boundary conditions are set as shown in Figure 3. The cylinder surface and the bottom surface are no-slip wall. The inlet is set as the velocity inlet. The outlet of the flow field is set as the pressure outlet, and the relative pressure is $0 \mathrm{~Pa}$. The top surface is set as symmetrical boundary conditions. The two sides of the flow field are set as free slip walls. The time step is $2 \mathrm{e}-5$ second. The water density is set as $1000 \mathrm{~kg} / \mathrm{m} 3$.

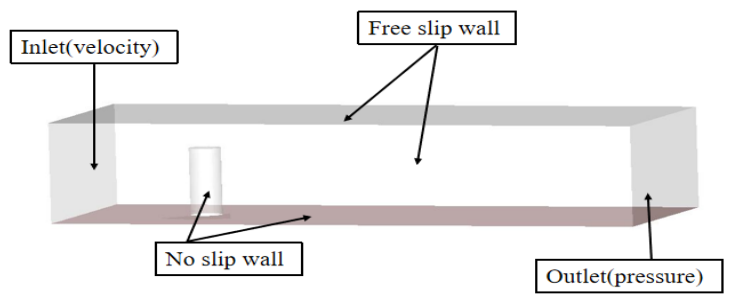

Fig. 3. Boundary condition.

\subsubsection{Meshing}

As shown in Figure 4, hexahedron elements are used for flow noise analysis, and O-type grids are used for boundary of cylindrical model to ensure boundary layer division. In order to ensure $y+=1$ of cylinder surface, the thickness of the first layer grid on the model surface is taken as $0.00075 \mathrm{D}$, and the growth factor is 1.09 . Considering the aspect ratio of the grid, the grid size of the cylinder spanwise is $0.004 \mathrm{D}$, and the grid is gradually sparse from the top of the cylinder to the upper boundary of the outer flow filed.The maximum size of the grid is $0.3 \mathrm{D}$, the number of grid nodes is 10.27 million, and the grid quality is above 0.8 .

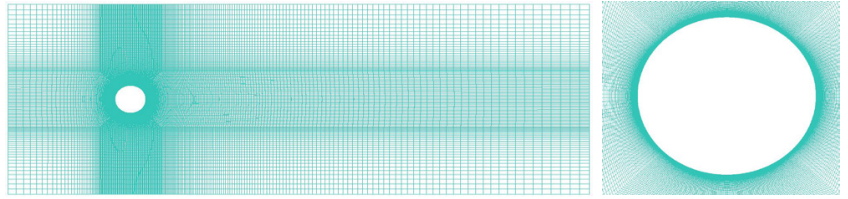

$\begin{array}{lll}\text { (a) } \mathrm{X}-\mathrm{Y} \text { plane grid } \quad(\mathrm{Z}=0.5 \mathrm{~h}) & \text { (b) Partial view of circumference }\end{array}$

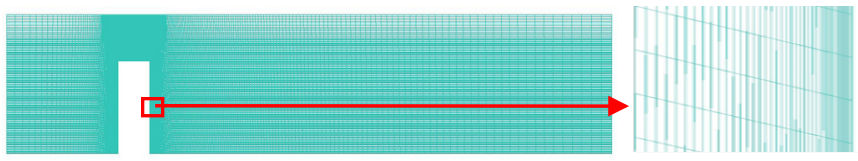

(c) $\mathrm{X}$-z plane grid $(\mathrm{Y}=0)$

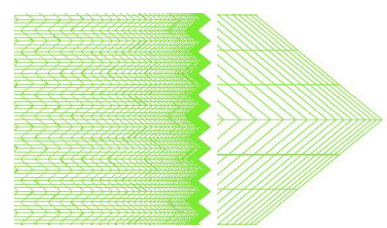

(d) V-shaped cylindrical

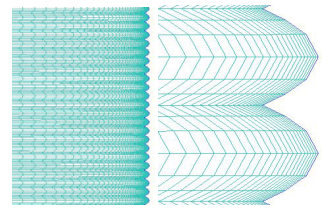

(e) U-shaped cylindrical

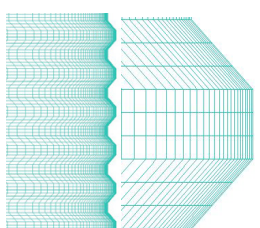

(f) Ladder shaped cylinder

Fig. 4. Computational flow field grid. 


\subsection{Sound field calculation}

\subsubsection{Sound field calculation model}

The sound field calculation model is given in Figure 5. The red area is the sound source domain.The gray region is the sound propagation domain. The upper surface of the sound propagation domain is set as infinite element, which is used to simulate the non reflecting boundary at infinite distance. The cylinder surfaces are total reflection rigid wall. The surface where the propagation domain coincides with the sound source domain is the coupling surface.

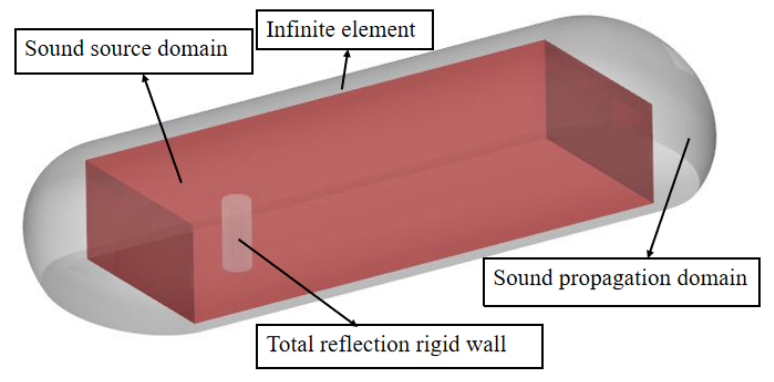

Fig. 5. Sound field calculation model.

\subsubsection{Monitoring points}

Three monitoring points are set at $50 \mathrm{~m}$ away from the center of cylinder bottom in three directions, namely A1 (625D, 0, 0.1), A2 (0,625D, 0.1), A3 $(0,0,625 \mathrm{D})$. The layout of monitoring points is shown in Figure 6.
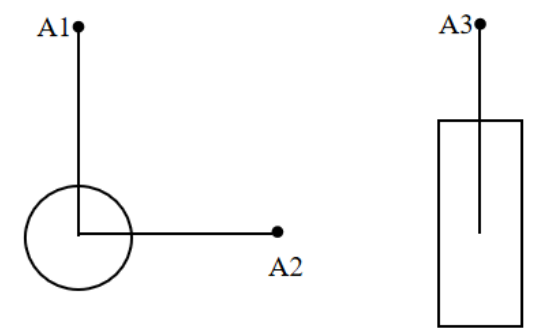

Fig. 6. Sound field monitoring point.

\subsubsection{Acoustic grids}

The basic requirement of acoustic computation is that there are at least six acoustic grids in one wavelength. In order to make the results of acoustic calculation accurate, the sound velocity in water at room temperature is usually $1500 \mathrm{~m} / \mathrm{s}$, and the frequency of sound pressure to be calculated in this paper is $500 \mathrm{~Hz}$, so the maximum size of grid should be less than $500 \mathrm{~mm}$. Since the source domain grid has been given in fluid calculation, only the propagation domain grid is shown in Figure 7. 


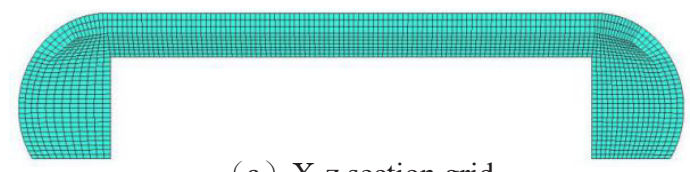

(a) X-z section grid

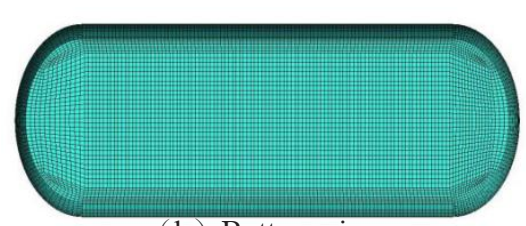

(b) Bottom view

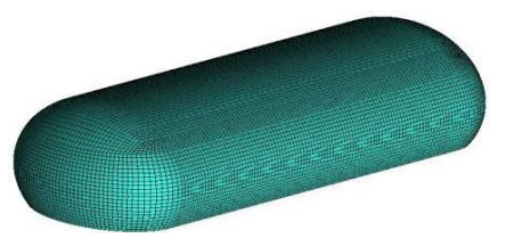

(c) Axonometric view

Fig. 7. Sound field grid.

\section{Analysis of sound field results}

\subsection{Acoustic results of smooth and bionic cylinders}

Table 1-3 show the total sound pressure level of smooth cylinder and bionic cylinders at monitoring points $\mathrm{A} 1, \mathrm{~A} 2, \mathrm{~A} 3$. The mean value is the average value of total sound pressure level of three points A1,A2,A3. The noise reduction amount is the difference of total sound pressure level between bionic cylinder and smooth cylinder at monitoring points. It can be seen that compared with smooth cylinder, V-shaped and U-shaped bionic cylinder have noise reduction effect, and ladder shaped cylinder can increase noise when Reynolds number is $3.99 \mathrm{e} 4$. The noise reduction of V-shaped bionic cylinder is the largest, which is $7.32 \mathrm{~dB}$, and the noise reduction effect is the best, followed by U-shaped cylinder and finally ladder shaped cylinder.

Table 1. Total sound pressure level of smooth cylinder and bionic cylinders $(\operatorname{Re}=3.99 \mathrm{e} 4)$.

\begin{tabular}{cccccc}
\hline & A1( $(\mathbf{d B})$ & $\mathbf{A 2}(\mathbf{d B})$ & $\mathbf{A 3}(\mathbf{d B})$ & mean value & Noise reduction \\
\hline Smooth & 73.83 & 73.88 & 73.62 & 73.78 & - \\
V & 67.44 & 67.40 & 67.72 & 67.52 & 6.26 \\
U & 68.64 & 68.62 & 68.54 & 68.60 & 5.18 \\
Ladder shape & 79.31 & 79.29 & 79.25 & 79.28 & -5.50 \\
\hline
\end{tabular}

Table 2. Total sound pressure level of smooth cylinder and bionic cylinders $(\operatorname{Re}=2.39 \mathrm{e} 5)$.

\begin{tabular}{cccccc}
\hline & $\mathbf{A 1}(\mathbf{d B})$ & $\mathbf{A 2}(\mathbf{d B})$ & $\mathbf{A 3}(\mathbf{d B})$ & mean value & Noise reduction \\
\hline Smooth & 108.23 & 108.35 & 108.20 & 108.26 & $\overline{7.32}$ \\
V & 100.96 & 100.93 & 100.93 & 100.94 & 6.75 \\
U & 101.52 & 101.48 & 101.53 & 101.51 & 3.03 \\
\hline Ladder shape & 105.25 & 105.19 & 105.24 & 105.23 & \\
\hline
\end{tabular}

Table 3. Total sound pressure level of smooth cylinder and bionic cylinders $(\operatorname{Re}=3.99 \mathrm{e} 5)$.

\begin{tabular}{cccccc}
\hline & $\mathbf{A 1}(\mathbf{d B})$ & $\mathbf{A 2}(\mathbf{d B})$ & $\mathbf{A 3}(\mathbf{d B})$ & mean value & Noise reduction \\
\hline Smooth & 148.28 & 148.23 & 148.37 & 148.29 & - \\
V & 142.64 & 142.62 & 142.65 & 142.64 & 5.65 \\
U & 144.36 & 144.35 & 144.36 & 144.36 & 3.93 \\
Ladder shape & 145.75 & 145.72 & 145.73 & 145.73 & 2.56 \\
\hline
\end{tabular}


The sound pressure level spectrums of smooth cylinder and bionic cylinders at monitoring point $\mathrm{A} 1$ are given respectively when $\mathrm{Re}=3.99 \mathrm{e} 4, \mathrm{Re}=2.39 \mathrm{e} 5$ and $\mathrm{Re}=3.99 \mathrm{e} 5$ in Figure 8 . It can be seen from the figure that with the increase of frequency, the sound pressure level decrease gradually. The flow noise around the cylinder mainly concentrates in the low frequency band, so reducing the low frequency noise around the cylinder can quickly reduce the cylinder flow noise. In addition, with the increase of Reynolds number, the peak value and corresponding frequency of sound pressure level also increase. This is due to the increase of velocity, the shedding frequency of wake vortex around the cylinder increases, which means the frequency corresponding to the peak value of sound pressure level increases. In addition, the V-shaped bionic cylinder has the best noise reduction effect when $\mathrm{Re}=2.39 \mathrm{e}$, and the noise reduction amount is $7.32 \mathrm{~dB}$.

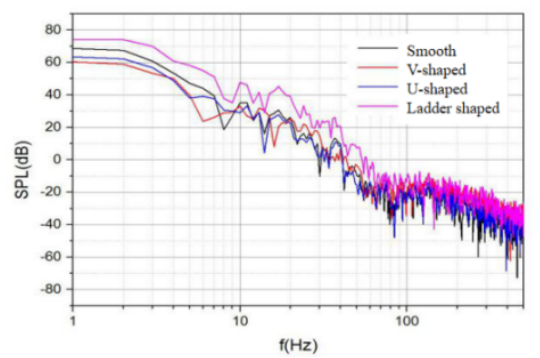

(a) $\mathrm{Re}=3.99 \times 10^{4}$

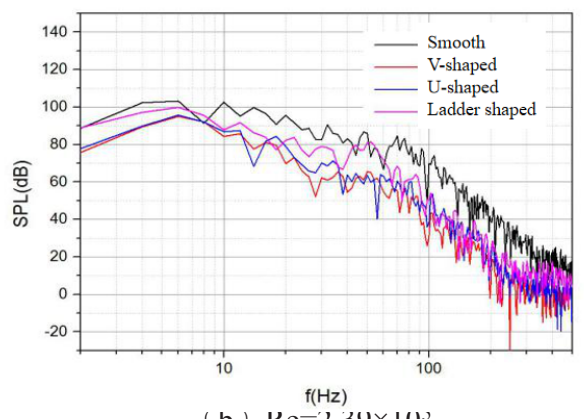

(b) $\mathrm{Ke}=2.3 \mathrm{y} \times 1 \mathrm{U}^{3}$

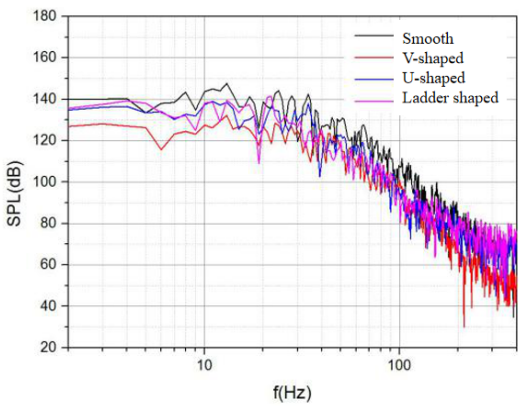

(c) $\mathrm{Ke}=3.9 \mathrm{Y} \times 1 \mathrm{U}^{\mathrm{J}}$

Fig. 8. Sound pressure level spectrum of smooth cylinder and bionic cylinder at monitoring point A1.

\subsection{Structure optimization}

\subsubsection{Basic model size}

Through the above analysis, it is concluded that the V-shaped cylinder structure can effectively reduce the flow noise around the cylinder. Therefore, the influence of V-shaped structure with different sizes on the flow noise is studied in this section. Suppose that the height of $\mathrm{V}$-shaped structure is $\mathrm{h}=1 \mathrm{~mm}$ and the width $\mathrm{s}$ is $\mathrm{h}, 2 \mathrm{~h}, 3 \mathrm{~h}, 4 \mathrm{~h}$ and $5 \mathrm{~h}$ respectively. As shown in Figure 9. 

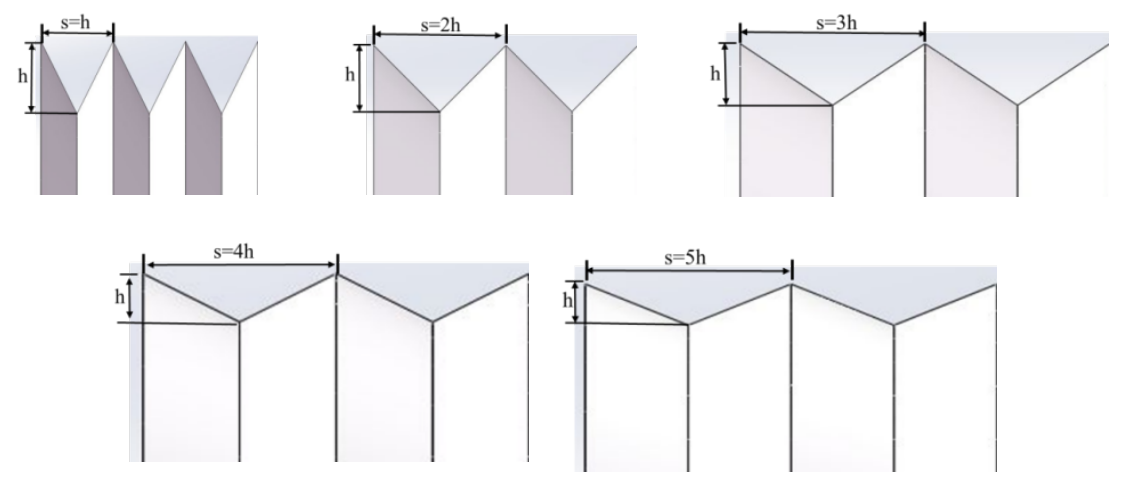

Fig. 9. V-shaped cylinder with different $\mathrm{S} / \mathrm{h}$ ratio.

\subsubsection{Comparison of acoustic results}

The total sound pressure levels of smooth cylinder and five V-shaped bionic structures with different $\mathrm{S} / \mathrm{h}$ at each monitoring points were compared. Six monitoring points were set up, which were perpendicular to the flow direction and were away from the center $h$ of the circle. $\mathrm{H}$ are $10 \mathrm{D}, 50 \mathrm{D}, 75 \mathrm{D}, 100 \mathrm{D}, 125 \mathrm{D}, 150 \mathrm{D}$, and the number of the six monitoring points are m1-m6. The schematic diagram of monitoring points is shown in Figure 10.

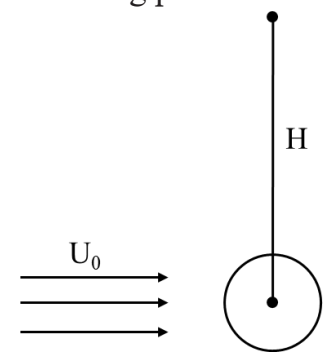

Fig. 10. Monitoring points.

Table 4 gives the total sound pressure level of smooth cylinder and V-shaped bionic cylinder with different $\mathrm{S} / \mathrm{h}$ at monitoring points $\mathrm{m} 1-\mathrm{m} 6$. It can be seen that the noise reduction effect of V-shaped bionic cylinder is related to $\mathrm{S} / \mathrm{h}$. When $\mathrm{S} / \mathrm{h}=1$ and $2, \mathrm{~V}$-shaped bionic cylinder has obvious noise reduction effect, and when $\mathrm{S} / \mathrm{h}=1$, the noise reduction effect is the best, and the noise reduction amount is about $12 \mathrm{~dB}$. When $\mathrm{S} / \mathrm{h}$ increases to 3 , the $\mathrm{V}$-shaped bionic cylinder can increase the noise on the contrary. When $\mathrm{s} / \mathrm{h}=4$ and 5 , the noise caused by the flow around the two cylinders is almost the same, and both of them increase the noise.

Table 4 .Total sound pressure level $(\mathrm{dB})$ at each monitoring point.

\begin{tabular}{ccccccc}
\hline & M1 & M2 & M3 & M4 & M5 & M6 \\
\hline Smooth cylinder & 156.67 & 129.85 & 123.90 & 120.09 & 117.37 & 115.29 \\
$\mathrm{~S}=\mathrm{h}$ & 144.90 & 117.52 & 111.05 & 107.93 & 105.64 & 103.94 \\
$\mathrm{~s}=2 \mathrm{~h}$ & 151.64 & 123.41 & 117.16 & 114.02 & 11.75 & 110.28 \\
$\mathrm{~s}=3 \mathrm{~h}$ & 159.36 & 132.91 & 126.55 & 122.38 & 119.62 & 117.51 \\
$\mathrm{~s}=4 \mathrm{~h}$ & 162.35 & 135.51 & 129.54 & 125.70 & 122.97 & 120.89 \\
$\mathrm{~s}=5 \mathrm{~h}$ & 162.00 & 135.09 & 129.09 & 125.22 & 122.48 & 120.38 \\
\hline
\end{tabular}

Figure 11 shows the noise reduction amount of V-shaped bionic cylinder with different $\mathrm{S} / \mathrm{h}$ at monitoring point M6, and the positive value indicates the noise reduction effect, and the negative value indicates the effect of increasing noise. It can be seen from the figure that 
the noise reduction effect of $\mathrm{V}$-shaped bionic cylinder has obvious relationship with $\mathrm{S} / \mathrm{h}$. With the increase of $\mathrm{S} / \mathrm{h}$, the noise increases gradually, and the critical value of $\mathrm{S} / \mathrm{h}$ is between 2 and 3.

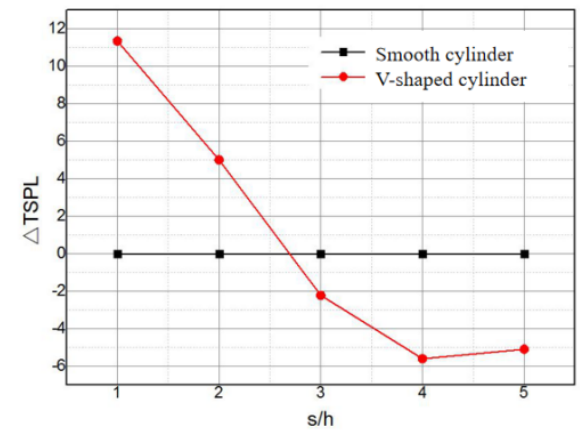

Fig. 11. Noise reduction of $\mathrm{V}$-shaped bionic cylinder with different $\mathrm{S} / \mathrm{h}$ at monitoring point M6.

The bionic V-shaped cylinder has the function of noise reduction, while the resistance can not be increased, so as to play the role of drag reduction and noise reduction.Table 3-2 shows the time average resistance coefficient and noise value of $\mathrm{V}$-shaped bionic cylinder with different $\mathrm{S} / \mathrm{h}$. If the drag reduction rate is positive, it means that it has the effect of drag reduction; if the drag reduction rate is negative, it has the effect of increasing resistance, and the noise reduction amount is the same.It can be seen from table 3-2 that the change trend of drag reduction and noise reduction of $\mathrm{V}$-shaped bionic cylinder with $\mathrm{S} / \mathrm{h}$ is basically the same. When $\mathrm{s} / \mathrm{h} \leqslant 2, \mathrm{~V}$-shaped bionic cylinder has drag reduction and noise reduction effect. When $\mathrm{s} / \mathrm{h} \geqslant 3, \mathrm{~V}$-shaped bionic cylinder can not reduce drag and noise, but increase drag and noise. The critical value of $\mathrm{S} / \mathrm{h}$ is between 2-3.

Table 5. Surface Comparison of total sound pressure level at each monitoring point between Vshaped bionic cylinder with 3-3 s/ $\mathrm{h}=2.5$ and $\mathrm{S} / \mathrm{h}=2$ and 3 (DB).

\begin{tabular}{ccccccc}
\hline & M1 & M2 & M3 & M4 & M5 & M6 \\
\hline $\mathrm{s}=2 \mathrm{~h}$ & 151.64 & 123.41 & 117.16 & 114.02 & 111.75 & 110.28 \\
\hline $\mathrm{s}=\mathbf{2 . 5 h}$ & $\mathbf{1 5 7 . 0 9}$ & $\mathbf{1 3 0 . 1 8}$ & $\mathbf{1 2 4 . 1 8}$ & $\mathbf{1 2 0 . 3 5}$ & $\mathbf{1 1 7 . 6 3}$ & $\mathbf{1 1 5 . 5 4}$ \\
\hline $\mathrm{s}=3 \mathrm{~h}$ & 159.36 & 132.91 & 126.55 & 122.38 & 119.62 & 117.51 \\
\hline
\end{tabular}

In conclusion, it can be seen that the noise reduction effect of $\mathrm{V}$-shaped bionic cylinder is approximately linear with $\mathrm{S} / \mathrm{h}$, and the noise reduction effect gradually decreases with the increase of $\mathrm{S} / \mathrm{h}$. Compared with smooth cylinder, the critical value of $\mathrm{S} / \mathrm{h}$ corresponding to the noise reduction effect of $\mathrm{V}$-shaped bionic cylinder is about 2.5 without increasing resistance. When $\mathrm{S} / \mathrm{h}<2.5, \mathrm{~V}$-shaped bionic cylinder has noise reduction effect; when $\mathrm{S} / \mathrm{h}>$ 2.5, V-shaped bionic cylinder does not have noise reduction effect; when $\mathrm{S} / \mathrm{h}$ increases to 45 , the noise tends to be stable.

\section{Conclusion}

In this paper, based on the transient calculation results of flow field, the acoustic characteristics of smooth cylinder and three typical bionic cylinder under different Reynolds number are discussed by using Lighthill acoustic analogy method, and the noise reduction mechanism of bionic cylinder is revealed. Finally, the noise reduction effect of V-shaped bionic cylinder with different size ratio is compared. 
(1) Among the three typical bionic cylinders, the V-shaped bionic cylinder has the best noise reduction effect.The $\mathrm{V}$-groove structure on the cylinder can slow down the shedding of wake, thus reducing the amplitude of pressure fluctuation caused by wake shedding, thus effectively reducing the dipole noise caused by the periodic shedding of wake.In addition, the V-shaped cylinder can prevent the transition from the separated shear layer on both sides of the cylinder to the turbulent flow, which reduces the vorticity. At the same time, the separation of the shear layer becomes more stable and the flow noise caused by vortex shedding is reduced.

(2) The noise reduction effect of V-shaped bionic cylinder is approximately linear with $\mathrm{S}$ / h, and the noise reduction effect gradually decreases with the increase of S / h.Compared with smooth cylinder, the critical value of $\mathrm{S} / \mathrm{h}$ corresponding to the noise reduction effect of $\mathrm{V}$-shaped bionic cylinder is about 2.5. When $\mathrm{s} / \mathrm{h}<2.5, \mathrm{~V}$-shaped bionic cylinder has noise reduction effect. When $\mathrm{s} / \mathrm{h}>2.5$, V-shaped bionic cylinder has no noise reduction effect. When $\mathrm{s} / \mathrm{h}$ increases to $4-5$, the noise tends to be stable.

\section{References}

1. Choi K S. The wall-pressure fluctuations of modified turbulent boundary layer with riblets[M]//Turbulence Management and Relaminarisation. Springer, Berlin, Heidelberg, 1988: 149-160.

2. Park S R, Wallace J M. Flow alteration and drag reduction by riblets in a turbulent boundary layer[J]. AIAA journal, 1994, 32(1): 31-38.

3. Graham, R. R. The Silent Flight of Owls[J]. Journal of the Royal Aeronautical Society, 1934, 38(286):837-843.

4. Soderman P T . Leading edge serrations which reduce the noise of low-speed rotors[J]. 1973.

5. Chen K, Liu Q, Liao G, et al. The sound suppression characteristics of wing feather of owl (Bubo bubo)[J]. Journal of Bionic Engineering, 2012, 9(2): 192-199.

6. Liu F, Lee K M, Yang C J. Hydrodynamics of an undulating fin for a wave-like locomotion system design[J]. IEEE/ASME Transactions on Mechatronics, 2012, 17(3): 554-562. 